\title{
ABSTRACTS
}

\section{DEVELOPMENT AND MODIFICATION OF \\ SOCIEMPATHY IN CLASS SOCIETY}

-New Direction of Sociometry in Educational Psychology-

\author{
By Tanaka, Kumajiro \\ Tokyo Gakugei University
}

(1) Sociometry has now rush into the age of revolutionary sociometry. "Sociempathic measurement" is advocated as a trial of such direction. According to the research by this instrument, development of sociempathy in pupils seems to be indicated by relative variation ( $V$ ). In a fer words, the relative variation is the less, the more promotion in grade.

(2) The results of sociempathic measurement are indicated by rank order of sociempathic status score. This rank order is modified by promotion or regrouping of class.

(3) The results of sociempathic measurement are figured in sociempathic-diagram. According to this diagram, sociempathic relation is modified by criteria and by promotion.
(4) It is necessary for us to consider the theoretical types of sociempathic relation when we examine the sociempathic-diagram. These types are classified as I-XIX types. The sociempathic patterns of the individual are signified by a sequence of modification in each criterion.

Sociempathy is developed and modified by perception, learning, or experiences, therefore it could be improved through training, guidance or therapy. We would like to contribute more or less to the advancement of theory and practice of guidance and therapy in educational field, clarifying the structure of social atom by the detailed analyses as the mentioned above.

\section{EFFECTS OF VARIOUS METHODS OF RETURNING TEST-PAPEIRS UPON ACHIEVEMENT OF PUPILS}

\author{
By Hashimoto, Juji \\ Yokohama National University
}

When we consider educational evaluation as an integral part of the effective learning situations, how to handle test-results, such as testscoring or methods of handing back test'spapers to pupils, will be one of the important problems. In this study, I focussed my attention exclusively to the influence of methods of handing back test-papers to pupils.

Three purposes of this study are as follows : 